ТЕХНОЛОГИЯ ИСКУССТВЕННОГО ИНТЕЛЛЕКТА ДЛЯ РАСПОЗНАВАНИЯ КОМПРЕССИОННЫХ ПЕРЕЛОМОВ ПОЗВОНКОВ С ПОМОЩЬЮ МОДЕЛИ МОРФОМЕТРИЧЕСКОГО АНАЛИЗА, ОСНОВАННОЙ НА СВЕРТОЧНЫХ НЕЙРОННЫХ СЕТЯХ

( ) А.В. Петряйкин ${ }^{1}$, Ж.Е. Белая ${ }^{2}$, А.Н. Киселева ${ }^{3}$, 3.Р. Артюкова ${ }^{1 *}$, М.Г. Беляев ${ }^{4}$, В.А. Кондратенко ${ }^{4}$, М.Е. Писов ${ }^{4,5}$, А.В. Соловьёв ${ }^{6}$, А.К. Сморчкова7, Л.Р. Абуладзе ${ }^{8}$, И.Н. Киева ${ }^{9}$, В.А. Феданов ${ }^{7}$, Л.Р. Яссин ${ }^{8}$, Д.С. Семенов ${ }^{1}$, Н.Д. Кудрявцев ${ }^{2}$, С.П. Щелыкалина ${ }^{10}$, В.В. Зинченко', Е.С. Ахмад' ${ }^{1}$, К.А. Сергунова', В.А. Гомболевский', Л.А. Низовцова', А.В. Владзимирский ${ }^{1}$, С.П. Морозов ${ }^{1}$

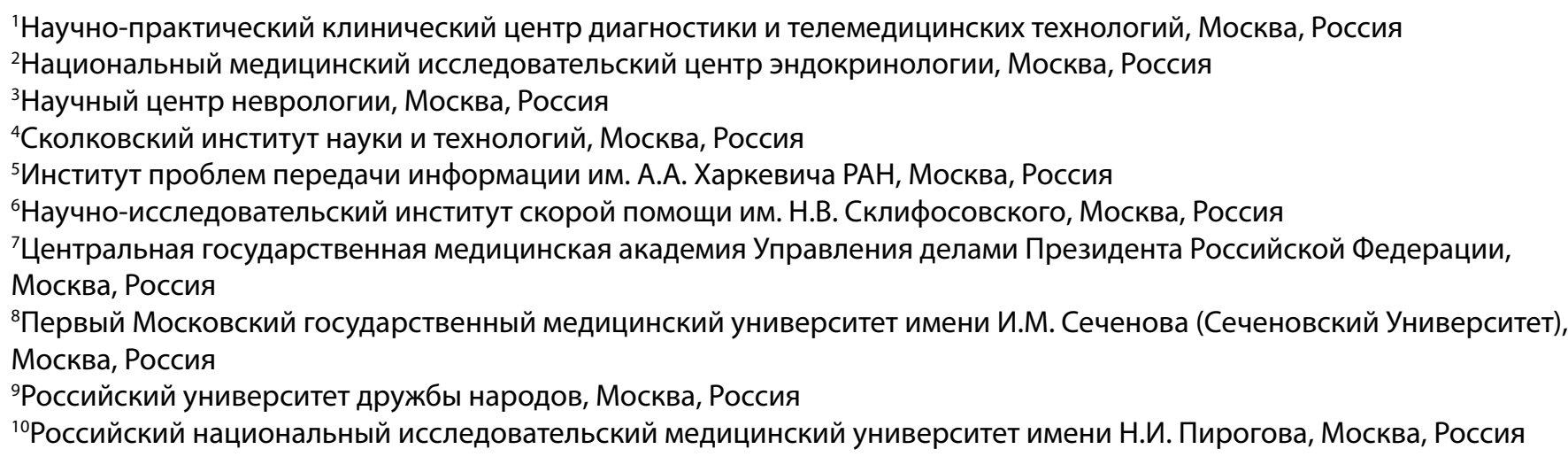

ОБОСНОВАНИЕ. Патологические низкоэнергетические (НЭ) компрессионные переломы (КП) тел позвонков являются признаками тяжелого течения остеопороза и предикторами последующих НЭ-переломов. До 84\% случаев КП тел позвонков корректно не описываются при рентгеновских методах, что обуславливает необходимость разработки ассистента радиолога на основе технологий искусственного интеллекта (ИИ) для повышения качества диагностики остеопороза, его осложнений и профилактики последующих переломов.

ЦЕЛЬ. Разработать модель ИИ для автоматической диагностики КП позвонков по данным компьютерной томографии (КТ) органов грудной клетки.

МЕтОДЫ. С сентября 2019 г. по май 2020 г. было проведено ретроспективное выборочное исследование, основанное на результатах КТ органов грудной клетки (ОГК). Были отобраны и анонимизированы результаты 160 КТ ОГК пациентов старше 60 лет, у 60\% из которых отмечались переломы с компрессией более 25\%. Разметка данных выполнена семью разметчиками. Проводились морфометрический анализ с вычислением средних размеров (вентрального, медиального, дорсального) тел позвонков и последующая полуколичественная классификация степени компрессии по Genant. Размеченный набор данных использовался для обучения созданной на основе двух сверточных нейронных сетей (CNN) модели ИИ Comprise-G, которая в последующем производила аналогичные измерения размеров тел позвонков с расчетом степени компрессии. Оценку модели выполняли по методу анализа ROC-кривых, расчета чувствительности и специфичности.

РЕЗУЛЬТАТЫ. Для 160 пациентов разметка проведена суммарно по 2066 позвонкам. Пациенты разделены на две группы: 100 - обучающая и 60 - тестовая выборка, не использованная в обучении модели ИИ. Оценка диагностической возможности Comprise-G: выявления 2-й и 3-й степени компрессии у пациентов при 5-FOLD кросс-валидации чувствительность 90,7\%, специфичность 90,7\%, площадь под ROC-кривой 0,974; для тестовых данных - чувствительность 83,2\%, специфичность 90,0\%, площадь под ROC-кривой 0,956; у позвонков для кросс-валидационных данных: чувствительность 91,5\%, специфичность 95,2\%, площадь под ROC-кривой 0,981; для тестовых данных: чувствительность 79,3\%, специфичность 98,7\%, площадь под ROC-кривой 0,978.

Вывод. Разработанная модель ИИ Comprise-G продемонстрировала высокие диагностические возможности для автоматического выявления КП тел позвонков при КТ-исследованиях грудной клетки и может быть рекомендована для дальнейшей валидации.

КЛЮЧЕВЫЕ СЛОВА: искусственный интеллект; Comprise-G; компрессионные переломы; остеопороз; компьютерная томография. 


\title{
ARTIFICIAL INTELLIGENCE FOR DIAGNOSIS OF VERTEBRAL COMPRESSION FRACTURES USING A MORPHOMETRIC ANALYSIS MODEL, BASED ON CONVOLUTIONAL NEURAL NETWORKS
}

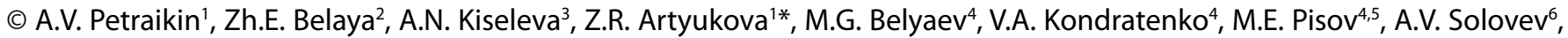 \\ A.K. Smorchkova ${ }^{7}$, L.R. Abuladze ${ }^{8}$, I.N. Kieva9 ${ }^{9}$, V.A. Fedanov ${ }^{7}$, L.R. Iassin ${ }^{8}$, D.S. Semenov ${ }^{1}$, N.D. Kudryavtsev ${ }^{1}$, S.P. Shchelykalina ${ }^{10}$, \\ V.V. Zinchenko', E.S. Akhmad' ${ }^{1}$, K.A. Sergunova' ${ }^{1}$, V.A. Gombolevskiy' ${ }^{1}$, L.A. Nizovtsova' ${ }^{1}$, A.V. Vladzymyrskyy' ${ }^{1}$, S.P. Morozov'

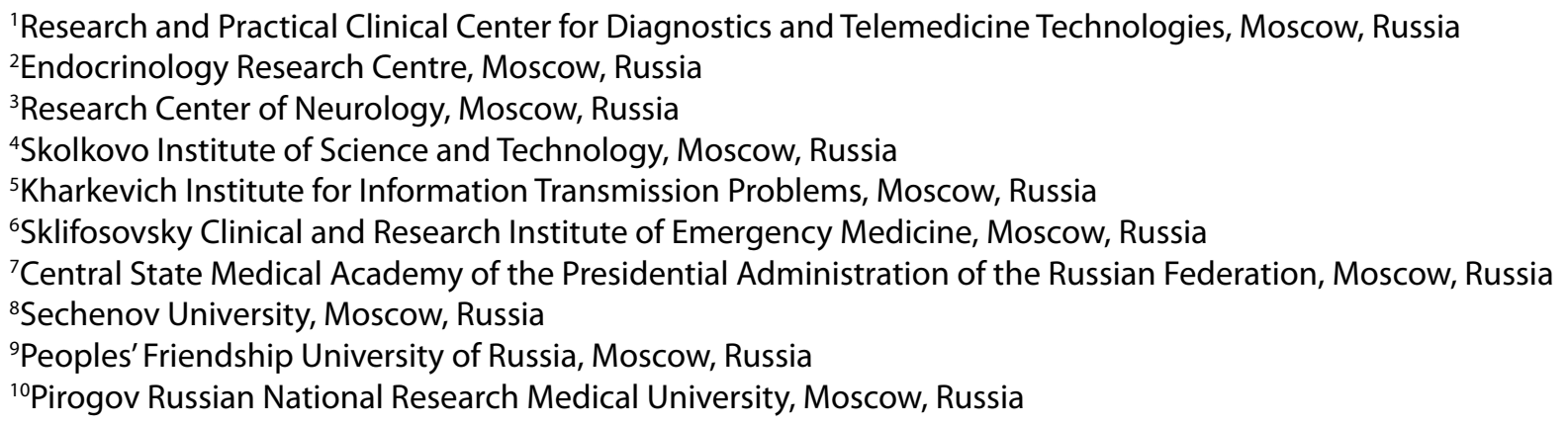

BACKGROUND: Pathological low-energy (LE) vertebral compression fractures (VFs) are common complications of osteoporosis and predictors of subsequent LE fractures. In $84 \%$ of cases, VFs are not reported on chest CT (CCT), which calls for the development of an artificial intelligence-based (Al) assistant that would help radiology specialists to improve the diagnosis of osteoporosis complications and prevent new LE fractures.

AIMS: To develop an Al model for automated diagnosis of compression fractures of the thoracic spine based on chest CT images.

MATERIALS AND METHODS: Between September 2019 and May 2020 the authors performed a retrospective sampling study of CCT images. The 160 of results were selected and anonymized. The data was labeled by seven readers. Using the morphometric analysis, the investigators received the following metric data: ventral, medial and dorsal dimensions. This was followed by a semiquantitative assessment of VFs degree. The data was used to develop the Comprise-G Al mode based on CNN, which subsequently measured the size of the vertebral bodies and then calculates the compression degree. The model was evaluated with the ROC curve analysis and by calculating sensitivity and specificity values.

RESULTS: Formed data consist of 160 patients (a training group - 100 patients; a test group - 60 patients). The total of 2,066 vertebrae was annotated. When detecting Grade 2 and 3 maximum VFs in patients the Comprise-G model demonstrated sensitivity - 90,7\%, specificity - 90,7\%, AUC ROC - 0.974 on the 5-FOLD cross-validation data of the training dataset; on the test data - sensitivity - 83,2\%, specificity - 90,0\%, AUC ROC - 0.956; in vertebrae demonstrated sensitivity - 91,5\%, specificity - 95,2\%, AUC ROC - 0.981 on the cross-validation data; for the test data sensitivity - 79,3\%, specificity - 98,7\%, AUC ROC - 0.978.

CONCLUSIONS: The Comprise-G model demonstrated high diagnostic capabilities in detecting the VFs on CCT images and can be recommended for further validation.

KEYWORDS: artificial intelligence; Comprise-G; vertebral fractures; osteoporosis; computed tomography.

\section{ОБОСНОВАНИЕ}

Остеопороз (ОП) - метаболическое заболевание скелета, характеризующееся снижением костной массы, нарушением микроархитектоники костной ткани и, как следствие, переломами при минимальной травме [1]. Распространенность ОП в Москве составляет 27\% среди мужчин и 34\% среди женщин старше 50 лет [2]. Более 2 млн жителей Европы имеют инвалидность по этому диагнозу [3]. Низкоэнергетические патологические переломы являются осложнением ОП и ведут к снижению качества жизни людей, инвалидности, летальным исходам и существенным экономическим затратам, достигающим 25 млрд рублей в год на лечение подобных переломов в России [2].

Компрессионные переломы (КП) тел позвонков следует выделить отдельно среди других низкоэнергетических переломов, поскольку в большинстве случаев они протекают бессимптомно и проявляются лишь в снижении роста и изменении осанки пациента [4]. Кроме того, данная патология недооценивается при описании рентгенограмм и изображений компьютерной томографии (КТ): только 13-16\% КП указываются врачами во время интерпретации КТ-данных [5, 6]. Между тем наличие КП позвонка формально означает диагноз ОП и повышает риск возникновения последующего КП в 5 раз, проксимального отдела бедра - в 2,8 раза и иных низкоэнергетических переломов - в 1,9 раза [7]. При своевременном выявлении КП дальнейшее развитие заболевания может быть предотвращено путем назначения антирезорбтивной и анаболической терапии [8].

Поэтому автоматическая диагностика КП позвонков с помощью моделей искусственного интеллекта (ИИ) может быть полезна в клинической практике для повышения доли выявленных КП. Многие из ранее разработанных подходов требуют предварительного обнаружения 
позвонков на КТ-изображениях $[9,10,11]$ или проведения сегментации позвоночника $[12,13,14]$. Однако даже при наличии высокой точности обнаружения КП разработанные классификаторы не позволяют количественно оценить степень компрессии позвонка.

Верификация КП при проведении КТ органов грудной клетки (ОГК) и брюшной полости является дополнительной нагрузкой на рентгенолога и, как правило, не проводится без специального назначения. Для автоматического выявления КП с использованием ИИ за рубежом были разработаны различные подходы, которые имеют как сильные стороны, так и ограничения. Часть методов, применяющих различные подходы ИИ, основана на определении вероятности КП отдельных позвонков у пациентов (например, более 50\% признается как достоверный признак наличия КП) $[11,14,15]$. Также применяется морфометрический анализ на основании разделения позвонка на 17 секторов [12], отличный от рекомендованного в базовой публикации [16], с полуколичественной классификацией степени компрессии. Однако в указанных работах не используются методы измерения высоты позвонков, соответствующие разметке при морфометрическом анализе [16].

Внедрение ИИ является национальной стратегией развития на период до 2030 г. (указ Президента от 10 октября 2019 г. № 490 «О развитии искусственного интеллекта в Российской Федерации») [17]. Развитие технологии ИИ для автоматической верификации пациентов с КП является актуальным для РФ.

\section{ЦЕЛЬ}

Разработать модель ИИ для автоматической диагностики КП позвонков по данным КТ ОГК.

\section{Дизайн исследования}

Было проведено ретроспективное выборочное исследование, основанное на результатах КТ ОГК. Схема проведенного исследования представлена на рисунке 1.

\section{Критерии соответствия}

Производился отбор пациентов от 60 лет, которым была выполнена КТ ОГК по стандартному протоколу сканирования со следующими параметрами: напряжение рентгеновской трубки при исследовании - 120 кВ; толщина срезов - 1,0 мм; фильтр реконструкции для мягких тканей; сила тока выбиралась автоматически с учетом анатомических параметров грудной клетки пациента (Sure Exposure 3D, 80-500 мA, обеспечивая качество изображения с условием: стандартное отклонение шума $10 \mathrm{HU})$. Время ротации трубки составляло 0,5 с; питч фактор составлял 0,828; алгоритмы итеративной реконструкции не использовались. Отбор пациентов производился до достижения сопоставимости количества пациентов с КП различных степеней в полученной выборке (табл. 1, 4), а также сбора необходимого объема данных для получения устойчивых результатов обучения разработанной модели. В соответствии с поставленной целью выборки пациентов содержали больше наблюдений с КП, чем среднепопуляционные показатели.

Критерии исключения: сколиоз III степени и выше с углом сколиотической деформации более $25^{\circ}$; несоответствие технологических режимов КТ-сканирования обозначенным выше; ошибки укладки пациента с отклонением оси позвоночника более $20^{\circ}$ от направления сканирования; наличие у пациента металлических имплантов позвоночника.

\section{Условия проведения}

Результаты КТ-сканирования были получены из базы Единого Радиологического Информационного Сервиса (ЕРИC), реализованной на платформе AGFA Enterprise v9.0. Томографические исследования выполняли в разных медицинских организациях с использованием оборудования одной модели (Aquillion 64), одного производителя (Canon Medical Systems, Japan).

\section{Продолжительность исследования}

Отбор результатов сканирования из ЕРИС проводился с сентября 2019 г. по февраль 2020 г. Процедура разметки выполнялась с октября 2019 г. по март 2020 г. Обработка полученных результатов и подготовка отчета - апрель-май 2020. Смещения запланированных временных результатов не было.

\section{Описание медицинского вмешательства}

В работе участвовали семь специалистов по разметке данных (разметчиков). Для разметки было использовано программное обеспечение NeuroML (разработчики - ИППИ РАН и «Сколтех», Россия) c web-доступом и персональными аккаунтами для каждого разметчика. Валидаторами разметки выступали рентгенологи со стажем работы 15 и 35 лет, которые согласовывали методику разметки, проверяли корректность аннотирования

\begin{tabular}{|c|c|c|c|c|}
\hline $\begin{array}{c}\text { Из базы данных } \\
\text { ЕРИС сформирована } \\
\text { анонимизированная } \\
\text { выборка пациентов } \\
60 \text { лет и старше, } \\
\text { прошедших КТ ОГК } \\
(n=160)\end{array}$ & $\begin{array}{c}\text { Семь специалистов } \\
\text { производили } \\
\text { разметку } \\
\text { вертикальных } \\
\text { размеров тел } \\
\text { позвонков } \\
\text { (вентральный, } \\
\text { срединный, } \\
\text { дорсальный) }\end{array}$ & $\begin{array}{c}\text { Разработан } \\
\text { двухэтапный } \\
\text { алгоритм } \\
\text { Comprise-G для } \\
\text { автоматической } \\
\text { разметки позвонков. } \\
100 \text { кТ исследований } \\
\text { включены } \\
\text { в обучающую и } 60 \text { - } \\
\text { в тестовую }\end{array}$ & $\begin{array}{c}\text { Оценка степени } \\
\text { компрессии } \\
\text { по классификации } \\
\text { Genant }\end{array}$ & $\begin{array}{c}\text { Сравнительный } \\
\text { анализ полученных } \\
\text { результатов } \\
\text { разметки } \\
\text { экспертами } \\
\text { и модели } \\
\text { Comprise-G }\end{array}$ \\
\hline
\end{tabular}

Рисунок 1. Схема проведенного исследования 


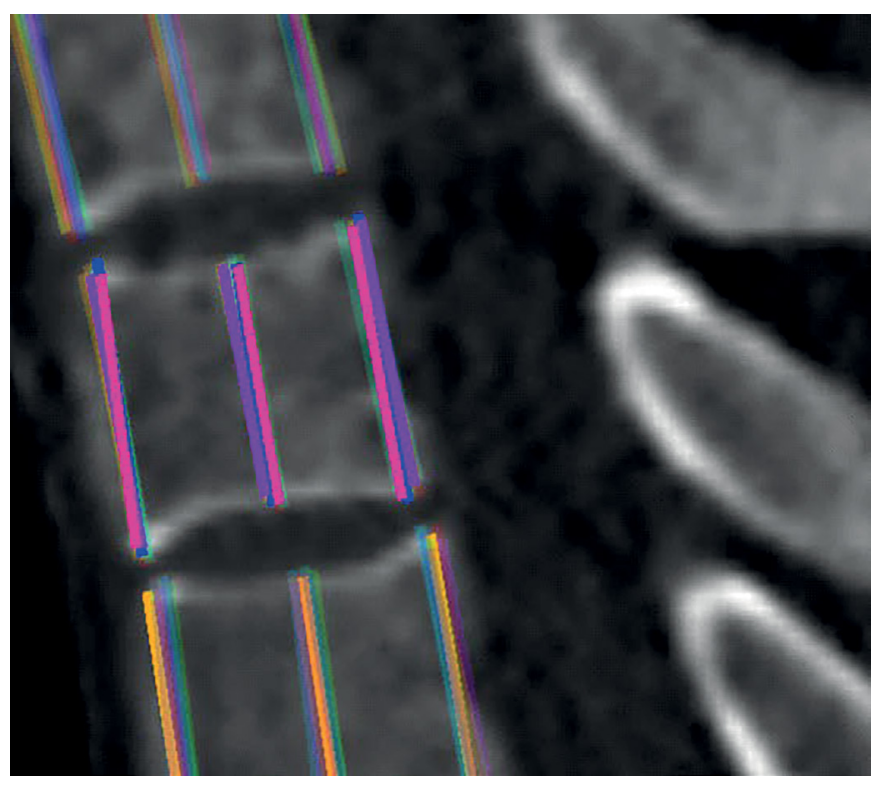

a

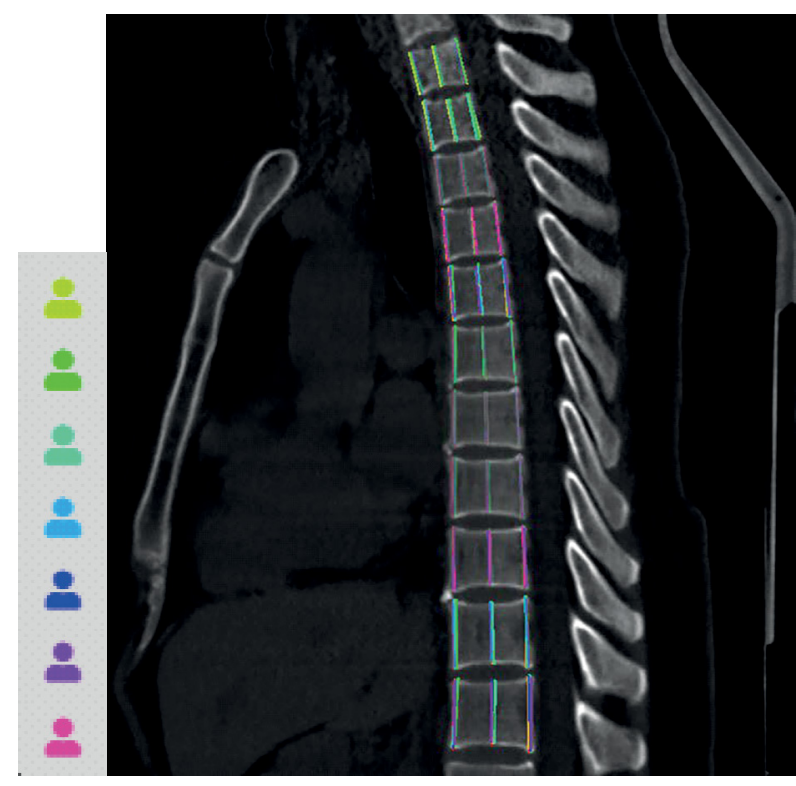

6

Рисунок 2. Пример разметки: а) разметка вентрального, медиального и дорсального размеров отдельного позвонка (ТhIl); б) Разметка грудного отдела позвоночника от Thl до ThXI. Приведены данные по семи разметчикам, маркированные цветом (интерфейс валидатора, представлены результаты всех разметок). Ярким цветом подсвечены измерения в плоскости реконструкции, более темные измерения в параллельных плоскостях, отражаемые проекционно.

перед отправкой данных в обучающую или тестовую выборку, а также проводили совместные консультации сложных для разметки случаев. Разметку выполняли на анонимизированных наборах данных слепым методом (разметчики видели только свои результаты). Средняя относительная погрешность измерений вертикальных и сагиттальных размеров в аннотаторе NeuroML составляла 0,02\% и 0,04\% соответственно. Проведены сравнения с измерениями в зарегистрированном ПО на платформе AGFA Enterprise v9.0: абсолютная погрешность определения линейных размеров составляла 0,5 мм (половина толщины среза реконструкции, 1,0 мм). Согласованность результатов измерений между 7 разметчиками, рассчитанная как средний коэффициент вариации по всем разметчикам, составила 3\%; средняя абсолютная погрешность измерений была равна 0,6 мм, что приближается к приборной погрешности 0,5 мм.

Разметка позвонков на КТ ОГК приводилась в сагиттальной проекции. Каждый из разметчиков отмечал высоту каждого позвонка в вентральном $\left(h_{a}\right)$, медиальном $\left(h_{m}\right)$, дорсальном $\left(h_{p}\right)$ отделах. При подсчете метрик в качестве истинных значений высот использовали средние по всем разметчикам величины. На основании этих данных рассчитывалась степень компрессии $\mathrm{G}$ (формула 1) каждого позвонка для классификации по Genant [13]. По результатам разметки и морфометрического анализа производилось сопоставление степени деформации с полуколичественной шкалой Genant, рекомендованной для этих целей международной организацией остеопороза - International Osteoporosis Foundation [18]. Степень 0 указывает на отсутствие перелома - снижение высоты позвонка менее чем на 20\%, степень 1 - минимальный перелом при уменьшении высоты в диапазоне 20-24\%, степень 2 - умеренный перелом с уменьшением высоты в пределах 25-40\% и степень 3 - тяжелый перелом с уменьшением высоты более чем на 40\% [19].
Разметка в аннотаторе NeuroML проводилась в единых параметрах, соответствующих «костному» окну: ширина $540 \mathrm{HU}$, центр $460 \mathrm{HU}$, толщина среза реконструкции 10 мм. Разметка каждого позвонка производилась на срезе с наибольшей шириной позвоночного канала, условно соответствующем середине тела позвонка. Разметку начинали с первого грудного позвонка до дистальных позвонков (Thl-ThXII/LII), полностью вошедших в область сканирования (рис. 2).

\section{Основной исход исследования}

Диагностические возможности метода Comprise-G для выявления пациентов с переломами тел позвонков G2, G3 при проведении рутинных КТ ОГК и первичная валидация этих данных.

\section{Дополнительные исходы исследования}

Диагностические возможности Comprise-G для классификации степени компрессии тел позвонков.

\section{Анализ в подгруппах}

Анализ в подгруппах производился по степени компрессии переломов тел позвонков.

\section{Методы регистрации исходов}

Для каждого позвонка на основании усредненных величин $\left(h_{a^{\prime}} h_{m^{\prime}} h_{p}\right)$ рассчитывали значения индекса $G$, количественно характеризующего степень компрессии позвонка:

$$
G=\frac{\max \left\{h_{a}, h_{m}, h_{p}\right\}-\min \left\{h_{a}, h_{m}, h_{p}\right\}}{\max \left\{h_{a}, h_{m}, h_{p}\right\}} * 100, \text { (1) }
$$

где $h_{a}$ - средняя высота переднего (вентрального) участка позвонка по всем разметчикам; $h_{m}-$ средняя высота срединного участка позвонка; $h_{p}$ - средняя высота дорсального участка позвонка. 
На основании полученного значения $G$ для каждого позвонка производилась классификация по Genant.

Проводилось прямое сравнение результатов оценки KT-изображений с применением Comprise-G и экспертной оценки. На основании этого проводился расчет площади под кривой операционных характеристик (AUC), определялись чувствительность и специфичность с расчетом доверительных интервалов.

\section{Этическая экспертиза}

Было проведено этическое рассмотрение данной работы на заседании ЛЭК «Морозовская ДГКБ ДЗМ», протокол № 160 от 11.08.2020 г.

\section{Статистический анализ}

Объемы выборки, необходимые для обучения разработанной модели автоматической детекции компрессионных переломов Comprise-G и отложенной выборки для оценки точности работы модели, определялись эмпирически по результатам предварительного обучения прототипа модели Comprise-G на 20 пациентах, которые впоследствии вошли в обучающую выборку.

Статистический анализ и визуализация полученных результатов осуществлялись на языке программирования Python 3. Для оценки диагностической значимости количественных данных, полученных моделью при морфометрическом анализе позвонков, применялся метод анализа ROC-кривых. C его помощью определялось оптимальное разделяющее значение количественного признака, позволяющее классифицировать позвонки и пациентов по степени компрессии, обладающее наилучшим сочетанием чувствительности и специфичности. Качество прогностической модели оценивалось исходя из значений площади под ROC-кривой с 95\% доверительным интервалом (ДИ).

\section{Алгоритм искусственного интеллекта}

Для автоматической оценки изменений формы позвонков по шкале Genant была разработана двухэтапная модель автоматической классификации степени компрессии тел позвонков Comprise-G.

Первый этап работы модели состоит в нахождении центров тел позвонков и «выпрямлении» позвоночника. За основу модели взята нейронная сеть с архитектурой, схожей с U-net [20] и 2D-soft-argmax-функцией в конце, позволяющей выделить координаты предсказанного центра (рис. 3 а, б). В качестве функции потерь используется средняя абсолютная ошибка, которая вычисляет среднюю сумму абсолютной разницы между истинными и прогнозируемыми значениями. Соединяя единой линией центры позвонков, формируется трехмерная кривая, перпендикулярно которой находятся плоскости позвонков (рис. 3 в). Отображение данных плоскостей параллельно друг другу позволяет получить «выпрямленный» позвоночник (рис. 3 г), центральный сагиттальный срез которого используется для проведения измерений высот на втором этапе (рис. 3 д). Простое выделение центральных срезов КТ-серии не могло быть использовано для пациентов с искривлениями позвоночника: данный алгоритм позволил включать в анализ все КТ-серии без исключения сложных случаев с выраженным кифозом и сколиозом. Обучение нейронной сети производилось с использованием оптимизатора Adam [21] со стандартными параметрами $(\beta 1=0,9, \beta 2=0,999)$ и скоростью обучения $\mathrm{Ir}=10^{-3}$ и заняло 10 ч на видеокарте Nvidia Tesla M40. Батч включал 3 KTсерии, было выполнено 10000 итераций до сходимости.

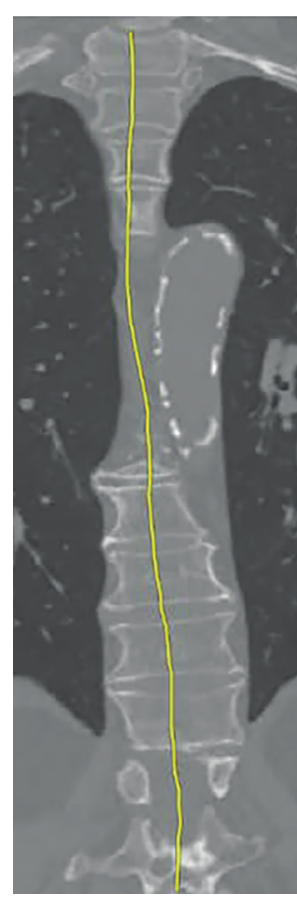

a

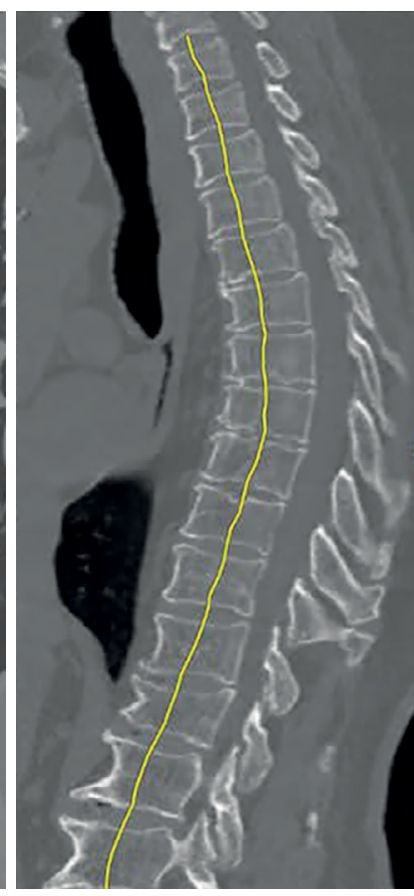

6

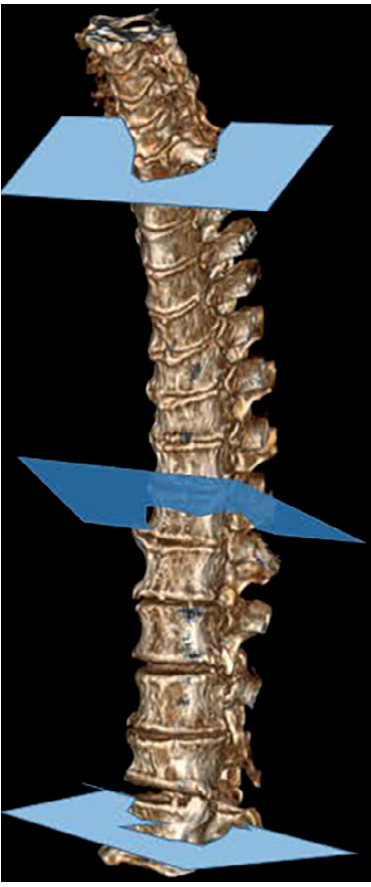

B

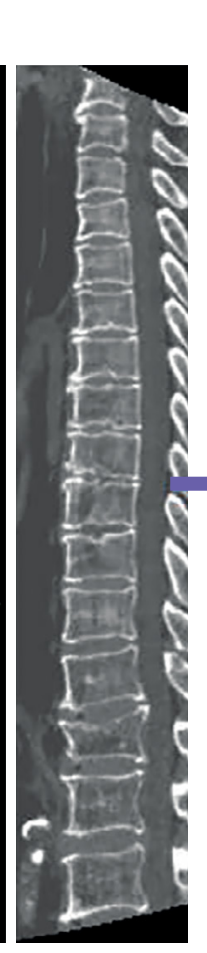

r
$G(\%) \quad$ (те- $G(\%) \quad$ (теРазмет- пень Модель пень
чики КП ИИ КП

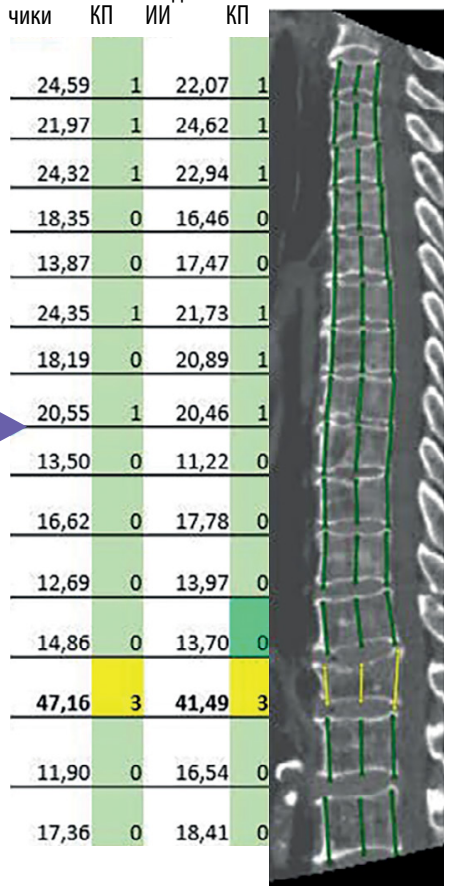

д

Рисунок 3. Обзор работы модели Comprise-G. Этап 1: локализация центров позвонков на 3D-KT а) в корональной; б) в сагиттальной плоскости; B) 3D-модель с непараллельными плоскостями, направленными перпендикулярно позвоночному столбу; г) создание 2D-изображения путем «выпрямления» позвоночника. Этап 2: д) определение ключевых точек и соответствующих высот тел позвонков, расчет индекса G: цветом обозначена степень компрессии позвонка по Genant (зеленый цвет — норма и слабая степень компрессии: 0; 1 , желтый — средняя и выраженная 2; 3). 
Таблица 1. Характеристики обучающей и отложенной тестовой выборок.

\begin{tabular}{lcccc}
\hline \multirow{2}{*}{ Выборка } & Количество & \multicolumn{2}{c}{ Возраст, лет } & \multicolumn{2}{c}{ Соотношение } \\
\cline { 3 - 4 } & пациентов & Медиана [квартили] & Размах [мин-макс] & мужчин/женщин \\
\hline Обучающая & 100 & $76[68 ; 82]$ & $60-92$ & $36 / 54$ \\
Отложенная, тестовая & 60 & $78[73 ; 82]$ & $62-91$ & $22 / 38$ \\
\hline
\end{tabular}

Второй этап - морфометрический анализ тел позвонков. Модель Comprise-G детектирует каждый позвонок с помощью сверточной нейронной сети, основанной на архитектуре YOLOv3 [22]. Далее на каждом позвонке размечает шесть точек, соответствующих вертикальным размерам тел позвонков (вентральному, медиальному и дорсальному). Использованная для обучения нейронной сети функция потерь складывается из ошибки детекции позвонка и суммы средних абсолютных ошибок для координат шести ключевых точек с учетом степени деформации позвонка: чем сильнее деформация, тем больший вклад вносит это слагаемое в функцию потерь. Обучение сети производилось в течение 4000 итераций с размером батча 30. Скорость обучения на начальных стадиях была уменьшена в 2 раза после 1000, 1400 и 2000 итераций с целью более быстрого достижения сходимости. Время обучения второй нейросети составило 40 ч на видеокарте Nvidia GTX 980. Принципиальная схема работы ИИ для морфометрического анализа тел позвонков с описанием отдельных этапов отражена на рисунке 3, подробно описана в статье [23].

\section{а) ROC-кривые для классификации позвонков}

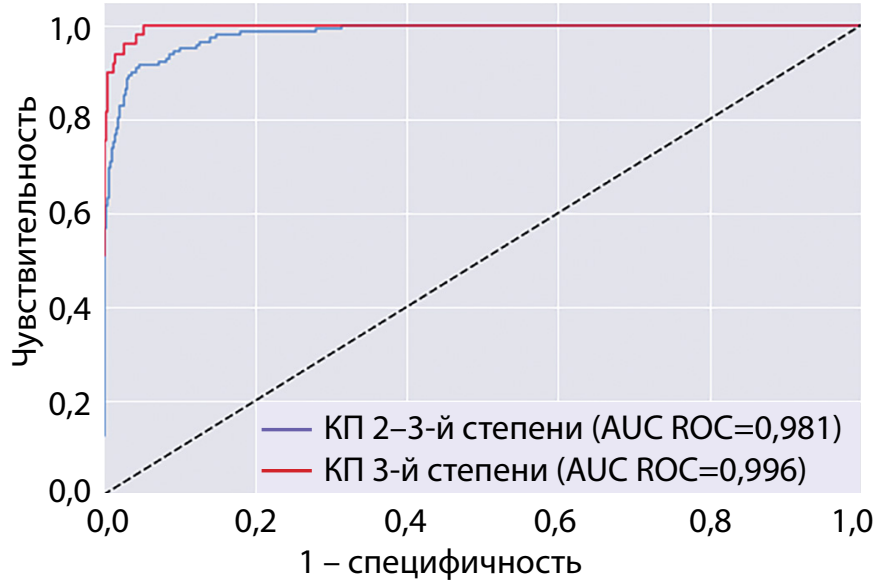

\section{РЕЗУЛЬТАТЫ}

Объекты (участники) исследования

Была сформирована выборка из КТ ОГК 160 пациентов. Медиана возраста пациентов составила 78 лет [25\% и 75\% квартили: 70; 82]. Все пациенты были разделены на обучающую и отложенную, тестирующую выборку, характеристики которых представлены в таблице 1.

Основные результаты исследования

Для оценки качества работы модели ИИ Comprise-G на кросс-валидационных данных проведен ROC-анализ для классификации отдельных позвонков и пациентов по степеням тяжести компрессионных переломовкоторый представлен на рисунке 4. Количественная характеристика полученных ROC-кривых (AUC ROC) с 95\% доверительными интервалами (ДИ) приведена в таблице 2. Чувствительность и специфичность бинарной классификации отдельных позвонков и пациентов с КП различной степени также приведены в таблице 2. При этом

\section{б) ROC-кривые для классификации пациентов}

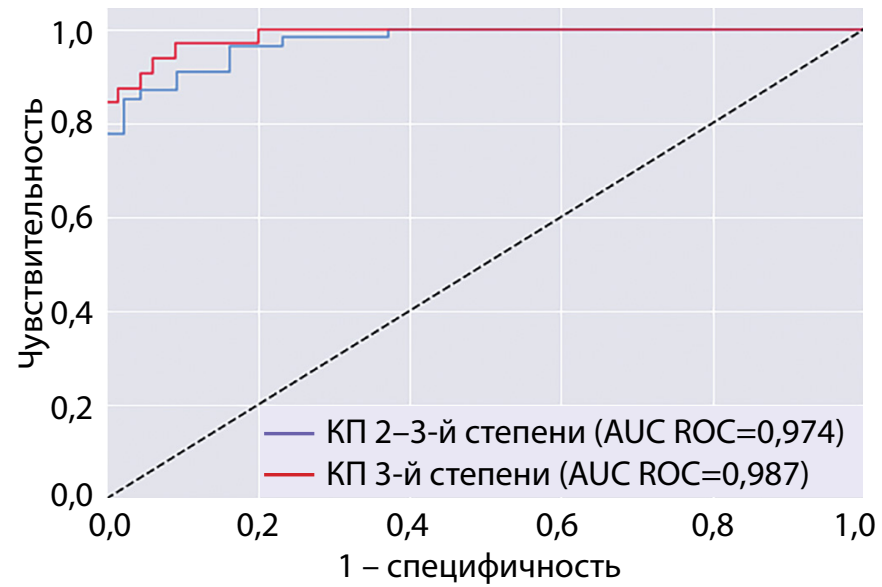

Рисунок 4. ROC-кривые для классификации позвонков (а) и пациентов (б) по степеням компрессии с помощью модели искусственного интеллекта Comprise-G по данным кросс-валидации ( $\mathrm{N}=1249$ позвонков, 100 пациентов)

Таблица 2. Площадь под ROC-кривой (AUC ROC), чувствительность и специфичность бинарной классификации компрессионных переломов, произведенной моделью Comprise-G на кросс-валидационных данных, сравнение с результатами экспертной разметки. В квадратных скобках обозначен 95\% доверительный интервал приведенных характеристик модели.

\begin{tabular}{lcc}
\hline \multicolumn{1}{c}{ Позвонки (N=1249) } & КП 2-3-й степени & КП 3-й степени \\
\hline ROC AUC & $0,981[0,973 ; 0,989]$ & $0,996[0,992 ; 1,0]$ \\
Чувствительность & $91,5[90,0 ; 93,0]$ & $98,0 \%[97,2 ; 98,8]$ \\
Специфичность & $95,2[94,0 ; 96,4]$ & $95,6 \%[94,5 ; 96,7]$ \\
\hline \multicolumn{1}{c}{ Пациенты (N=100) } & $0,974[0,943 ; 1,0]$ & $0,987[0,965 ; 1,0]$ \\
\hline ROC AUC & $90,7[83,4 ; 98,0]$ & $96,9 \%[92,5 ; 101,3]$ \\
Чувствительность & $90,7[83,4 ; 98,0]$ & $90,8 \%[83,5 ; 98,1]$ \\
Специфичность & &
\end{tabular}


a) ROC-кривые для классификации позвонков

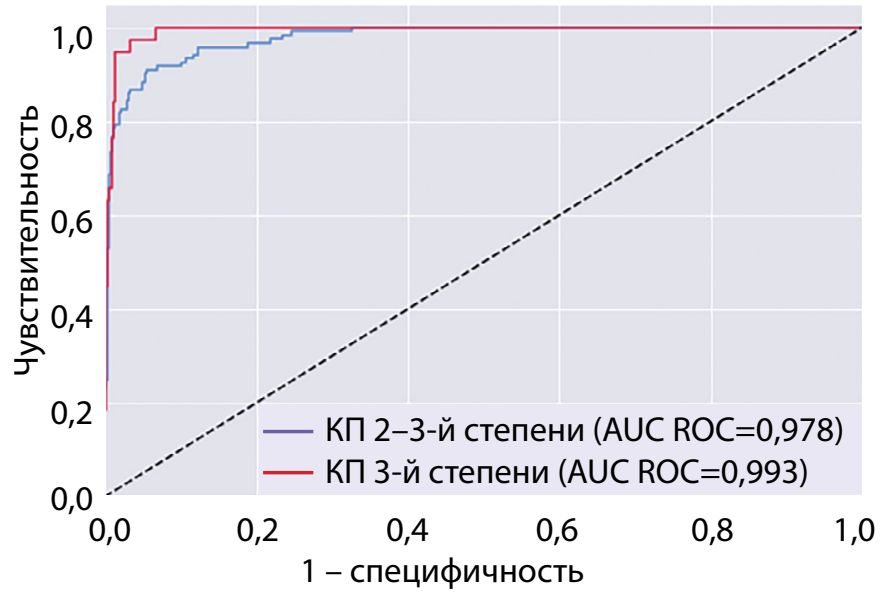

б) ROC-кривые для классификации пациентов

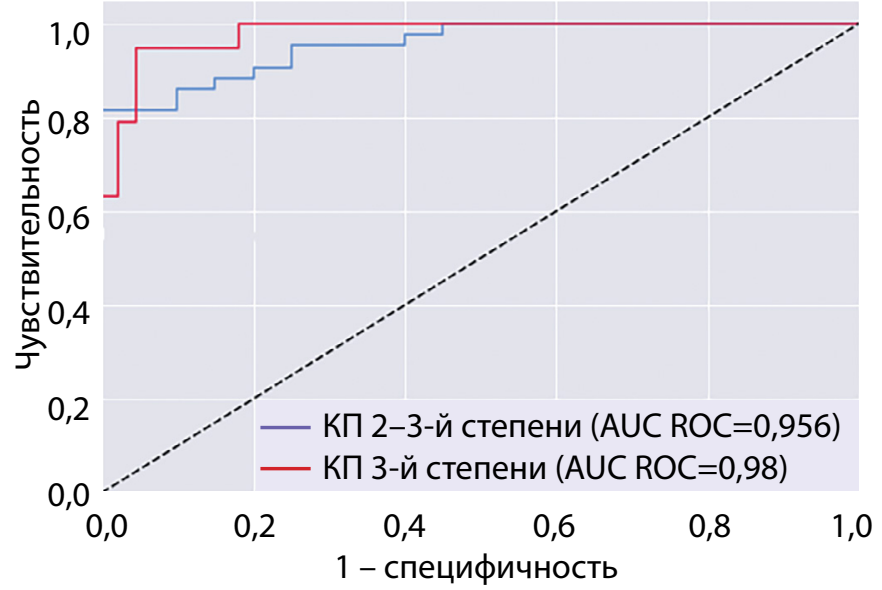

Рисунок 5. ROC-кривые для классификации позвонков (а) и пациентов (б) по степеням компрессии с помощью модели искусственного интеллекта Comprise-G при тестировании на отложенной выборке ( $\mathrm{N}=817$ позвонков, 60 пациентов).

Таблица 3. Площадь под ROC-кривой (AUC ROC), чувствительность и специфичность бинарной классификации компрессионных переломов, произведенной моделью Comprise-G на отложенной выборке. В квадратных скобках обозначен 95\% доверительный интервал приведенных характеристик модели.

\begin{tabular}{lcc}
\hline \multicolumn{1}{c}{ Позвонки (N=817) } & КП 2-3-й степени & КП 3-й степени \\
\hline ROC AUC & $0,978[0,968 ; 0,988]$ & $0,993[0,987 ; 0,999]$ \\
Чувствительность & $79,3[76,5 ; 82,1]$ & $97,4 \%[96,3 ; 98,5]$ \\
Специфичность & $98,7[97,9 ; 99,5]$ & $96,5 \%[95,2 ; 97,8]$ \\
\hline \multicolumn{1}{c}{ Пациенты (N=60) } & в второй степени & Третья степень \\
\hline ROC AUC & $0,956[0,904 ; 1,0]$ & $0,980[0,945 ; 1,0]$ \\
Чувствительность & $83,2[73,7 ; 92,7]$ & $94,7 \%[89 ; 100]$ \\
Специфичность & $90,0 \%[82,4 ; 97,6]$ & $95,5 \%[90,3 ; 100]$ \\
\hline
\end{tabular}

приведены данные для клинически значимых 2-й и 3-й степеней компрессионных переломов.

Данные ROC-кривых для отложенной выборки, в которую вошли данные по 60 пациентам, не использованные в обучении нейросети, представлены на рисунке 5. Количественная характеристика полученных ROC-кривых с 95\% ДИ для отложенной выборки приведена в таблице 3, где также приведены данные по чувствительности и специфичности бинарной классификации.
Таблицы сопряженности для классификации позвонков и пациентов по степеням компрессии, сопоставляющие экспертную классификацию с автоматической детекцией, произведенной моделью, приведены на рисунке 6. Классификация позвонков по степеням компрессии по Genant производится алгоритмом с точностью 81,5\% (95\% ДИ 79,8-83,2), классификация пациентов - с точностью 73,8\% (95\% ДИ 67,0-80,6]), причем наибольшей чувствительностью и положительной
Пациенты

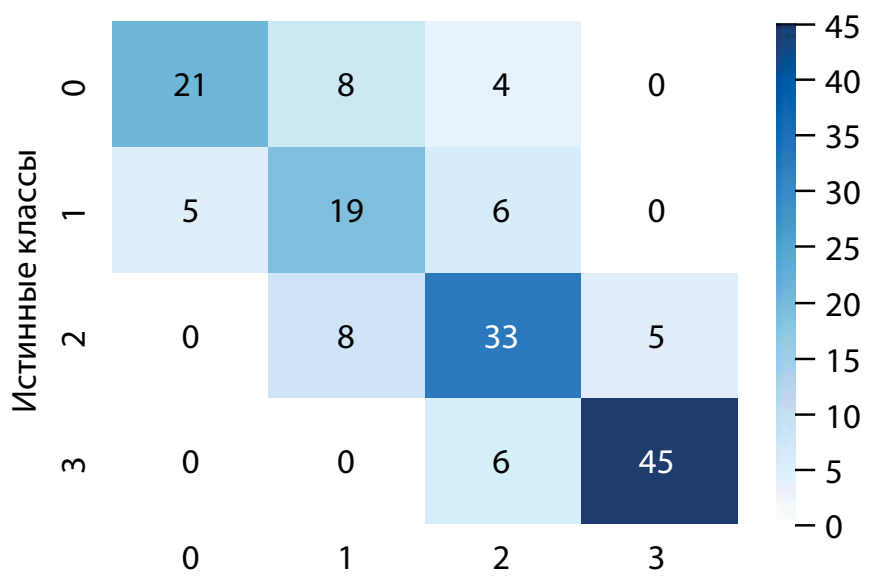

Предсказанные классы
Позвонки

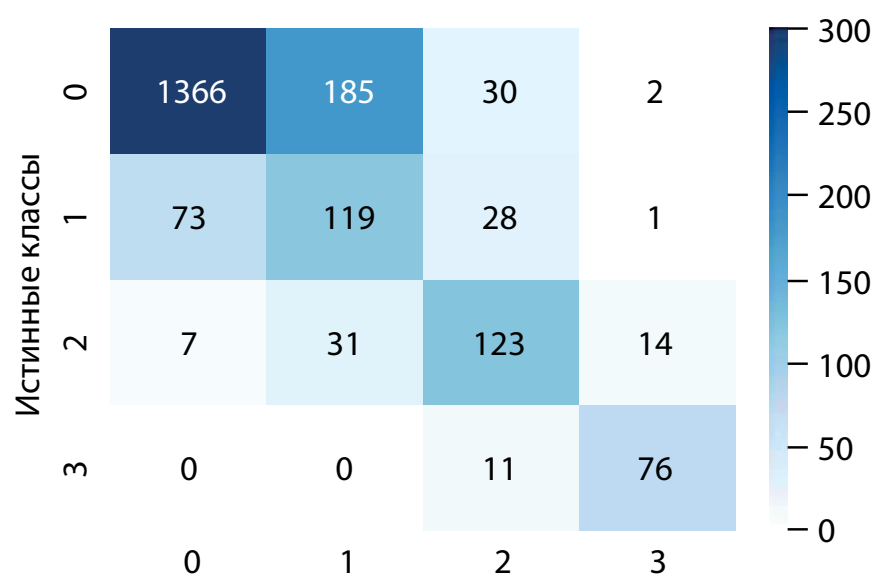

Предсказанные классы

6

Рисунок 6. Таблицы сопряженности классификации позвонков (а) и пациентов (б) по степеням компрессионных переломов, произведенной моделью Comprise-G. Строки - степени компрессии по данным экспертной разметки, столбцы — по измерениям модели 


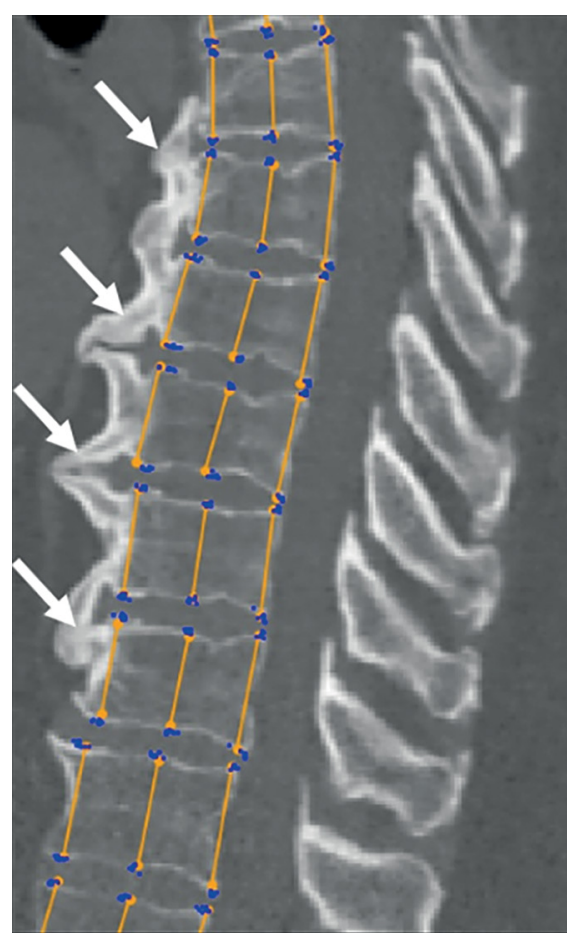

a

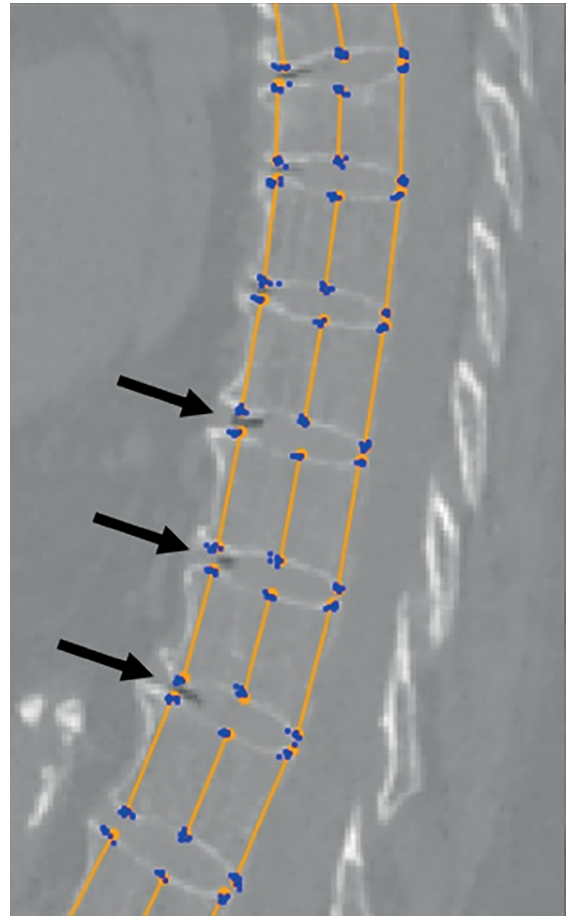

6

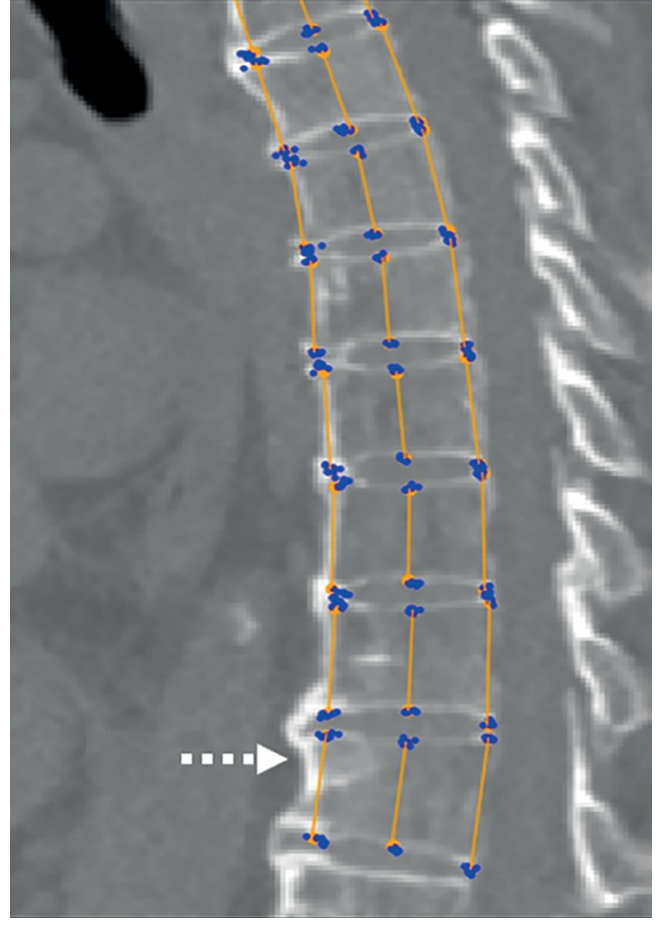

B

Рисунок 7. Примеры соответствия ручной разметки экспертами (синие точки - отдельные координаты по семи разметчикам) автоматическому измерению моделью Comprise-G (оранжевые отрезки) для трех пациентов. Белые стрелки (а) - выраженный спондилез с формированием замковых остеофитов, черные стрелки (б) - участки вакуум-феномена в межпозвоночных дисках, белая пунктирная стрелка (в) - остеофиты с участком склероза в теле позвонка. Модель демонстрирует устойчивость в данных сложных заданиях.

прогностической ценностью обладает определение 3-й и 2-й степеней компрессии.

В результате работы модели Comprise-G локализация позвонков в 3D-формате с последующей процедурой «выпрямления» (рис. 3 г, д) осуществляется со средней ошибкой в 1,0 мм. Сегментация отдельных позвонков в 2D достигла точности 0,99 и полноты 1,0.

Примеры автоматических измерений вертикальных размеров тел позвонков, произведенных нейронной сетью модели Comprise-G, представлены на рисунке 7.

\section{Дополнительные результаты исследования}

В результате проведения морфометрического анализа были определены классы степени максимальной деформации для отдельных позвонков и пациентов В соответствии с классификацией степени компрессии согласно полуколичественному подходу Genant $[15,18]$. Результаты представлены в таблице 4.

По результатам усреднения по 7 разметчикам линейных размеров был рассчитан индекс степени компрессии G для 2066 позвонков, среди которых 483 имели компрессионные переломы. Распределение количества переломов тел позвонков у отдельных пациентов представлено на рисунке 8. Выявленные по индексу G компрессионные переломы были впоследствии подтверждены двумя экспертами-рентгенологами.

Распределение позвонков с КП - бимодальное с локальными максимумами в среднегрудном отделе (локализации ThVI-VIII) и нижней части грудного отдела с переходом в поясничный отдел (ThXI-LI)
Таблица 4. Распределение позвонков и пациентов в результате выполненного морфометрического анализа и последующей классификации степени компрессии согласно полуколичественному подходу Genant $[15,18]$ (экспертная разметка), n (\%)

\begin{tabular}{ccc}
\hline $\begin{array}{c}\text { Степень } \\
\text { КП }\end{array}$ & $\begin{array}{c}\text { Количество } \\
\text { позвонков }\end{array}$ & $\begin{array}{c}\text { Количество } \\
\text { пациентов }\end{array}$ \\
\hline 0 & $1583(76,6)$ & $33(20,6)$ \\
1 & $221(10,7)$ & $30(18,8)$ \\
2 & $175(8,5)$ & $46(28,8)$ \\
3 & $87(4,2)$ & $51(31,9)$ \\
\hline
\end{tabular}

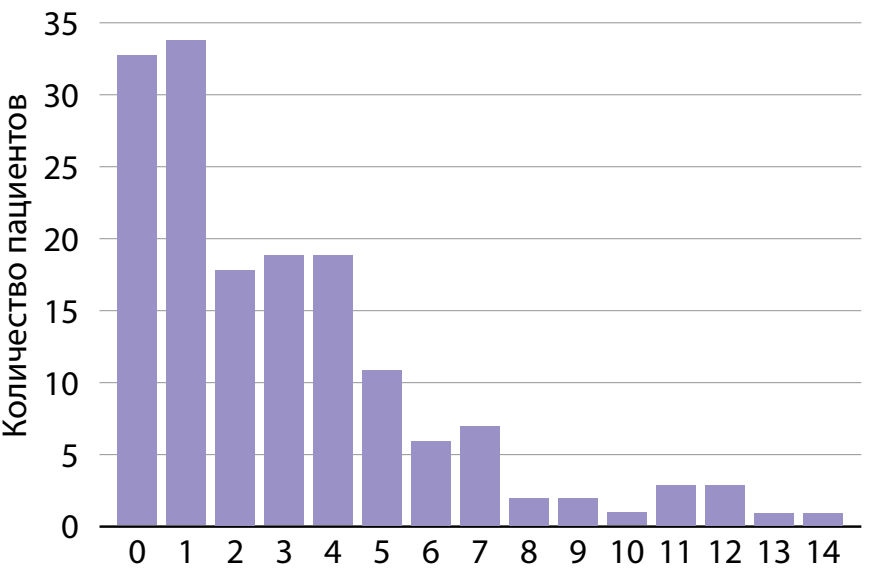

Количество переломов у одного пациента

Рисунок 8. Распределение пациентов по количеству переломов (всех степеней) согласно экспертной разметке $\mathrm{N}=160$. 
Распределение позвонков в грудном и поясничном отделах с компрессионными переломами

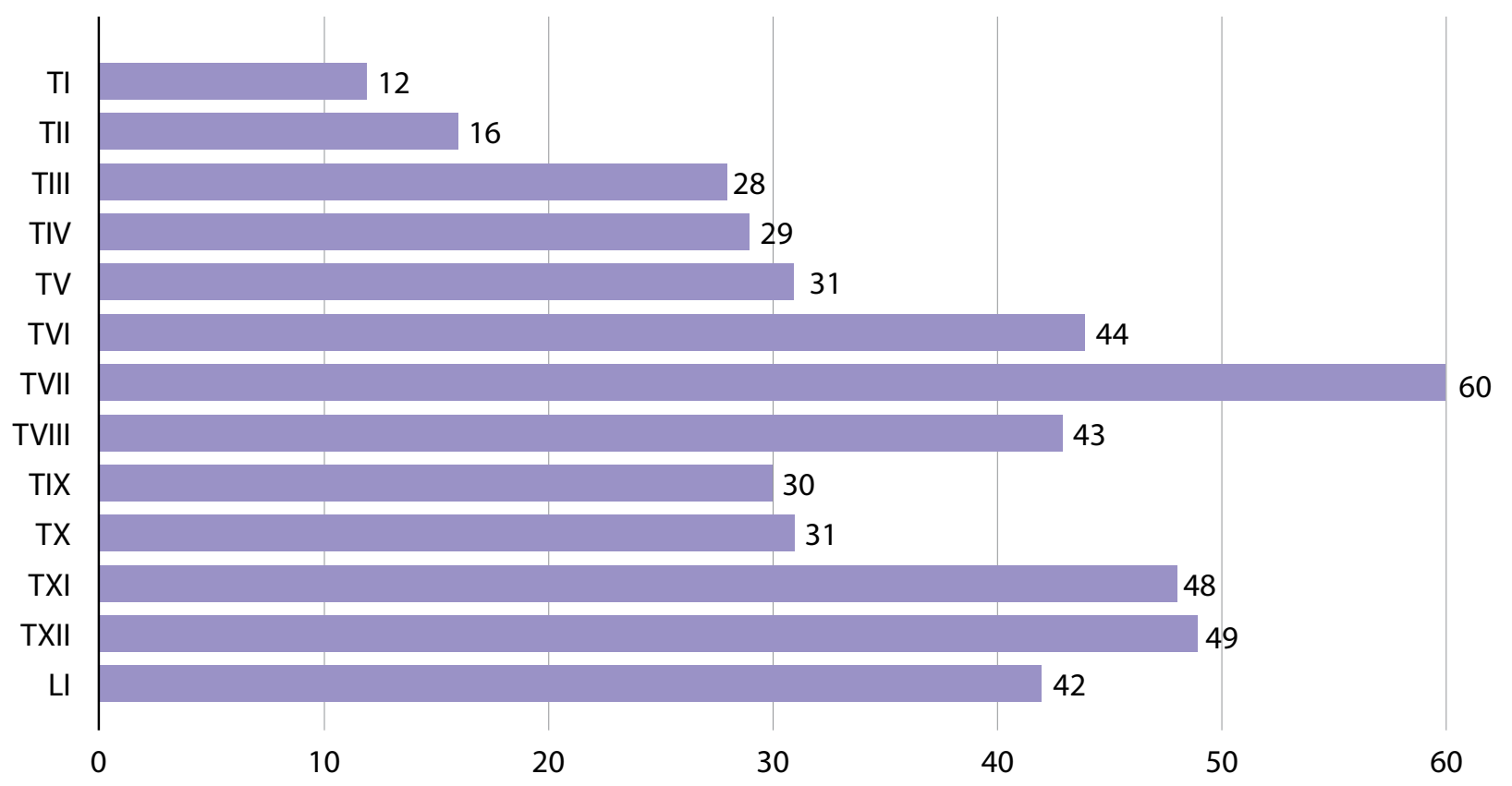

Количество случаев

Рисунок 9. Распределение количества позвонков с компрессионными переломами в грудном отделе позвоночника и первом поясничном позвонке (экспертная разметка), $\mathrm{N}=160$.

Таблица 5. Распределение позвонков по степени компрессии в ходе выполнения морфометрического анализа и последующей классификации степени компрессии согласно полуколичественному подходу Genant (экспертная разметка), n (\%)

\begin{tabular}{|c|c|c|c|c|}
\hline Тип компрессии & Все степени & 1-я степень & 2-я степень & 3-я степень \\
\hline $\begin{array}{l}\text { Срединная } \\
\text { деформация }\end{array}$ & $411(85)$ & $183(83)$ & $147(84)$ & $81(93)$ \\
\hline $\begin{array}{l}\text { Передняя } \\
\text { деформация }\end{array}$ & $72(15)$ & $38(17)$ & $28(16)$ & $6(7)$ \\
\hline
\end{tabular}

позвоночника (рис. 9). Самый распространенный тип деформации в выборке - срединная (максимальная разница между дорсальными и срединными размерами), значительно реже встречалась передняя; позвонки с деформацией, когда дорсальный размер был минимальным, при наборе данных отсутствовали (табл. 5). Согласованность между моделью и экспертами в определении формы КП составила 91,0\% (95\% ДИ 88,4-93,6).

\section{Нежелательные явления}

Нежелательные явления не отмечены, так как работа выполнена ретроспективно, без дополнительного вмешательства для пациента.

\section{ОБСУЖДЕНИЕ}

В ходе нашего исследования разработана модель морфометрического анализа тел позвонков, производящая автоматическую идентификацию, морфометрию и оценку степени тяжести КП позвонков с расчетом индекса G (соответствует классификации Genant), обладающая высокой чувствительностью и специфичностью.

Первый этап работы модели Comprise-G - новый метод идентификации центра позвоночного столба на трехмерных KT- данных и последующее приведение к «выпрямленному» двумерному средне-сагиттальному срезу для дальнейшего измерения параметров каждого позвонка. Это позволяет эффективно компенсировать основные виды деформации позвоночного столба естественные изгибы (кифоз/лордоз), сколиоз, торсионную деформацию. Подобный подход также реализован в Zebra [11, 14].

Второй этап работы модели Comprise-G производит сегментацию позвонков и находит шесть ключевых точек, позволяющих измерить три высоты (h) каждого позвонка, с последующей оценкой степени компрессии по Genant. На обоих этапах применяются сверточные нейронные сети, более подробно описанные в разделе «алгоритм искусственного интеллекта» и представленные в публикации [23]. При сравнении с результатами разметки экспертами показатели ROC AUC для кросс-валидационной и отложенной тестовой выборки превышают значение 90\% для всех степеней КП как для классификации отдельных позвонков, так и для классификации пациентов, что говорит о высокой эффективности полученной модели. ROC AUC возрастает с увеличением степени КП в обоих случаях.

Рассматриваемая задача распознавания отдельных позвонков и определение степени их компрессии при 
патологическом переломе потенциально решаема для моделей ИИ. Это обусловлено высоким контрастом костных структур, анатомическим «консерватизмом» исследуемого объекта - позвоночника, включающего сходные между собой элементы - тела позвонков, деформацию которых необходимо оценить.

В литературе описаны подходы автоматической детекции КП по результатам лучевых исследований разной модальности: КТ [11-14, 15], денситометрии в боковой проекции [24], магнитно-резонансной томографии. Технологии ИИ привлекательны не только для распознавания КП, но и для диагностики других патологических переломов, например, дистальной трети предплечья [25].

Наибольшие отклонения модели от истинных значений наблюдаются в случаях, где высока изменчивость экспертного мнения. Это в большей степени касается деформации позвонков с низкой, 1-й степенью компрессии (от 20 до 25\%). Для данных переломов затруднительно определить, насколько это состояние не является клиновидной деформацией, например, в среднегрудном отделе позвоночника. Неустойчивость модели при диагностике деформации 1-й степени подтверждается данными таблиц сопряженности (рис. 6), так, при 1-й степени компрессии корректно могли быть предсказаны только 54\% позвонков и 63\% пациентов (65\% позвонков, по данным J.E. Burns [12]). Для 2-й степени компрессии это 73\% позвонков и 71\% пациентов (83\% позвонков [12]), для 3-й степени - 87\% и 88\% (59\% позвонков [12]). Клинически значимые переломы 2-3-й степени компрессии детектируются с ROC AUC 97,8\% для позвонков и 95,6\% для пациентов при тестировании на отложенной выборке. В ряде публикаций также учитываются как клинически значимые КП 2-й и 3-й степени компрессии [26, 27]. Таким образом, для оценки работы модели Comprise-G мы привели данные по умеренным и тяжелым переломам.

Разработанная модель ИИ Comprise-G успешно детектирует размеры тел позвонков вблизи визуально «сложных» участков, где область измерения располагается вблизи участков повышенной плотности (спондилез обызвествление передней продольной связки с формированием остеофитов), вблизи участков сниженной плотности (при вакуум-феномене).

В ходе оценки эффективности работы модели Comprise-G получены данные ROC AUC, а также параметры чувствительности и специфичности. Чувствительность/специфичность модели Comprise-G составили 79,3\%/98,7\% при детекции переломов 2-й и 3-й степени компрессии по данным ROC-анализа на отложенной выборке. Это сопоставимо с результатами работы модели, основанной на рекуррентной сверточной сети (RCN), реализованной в коммерческом продукте Zebra (83,9\%/93,8\%) [14]. Несколько большая чувствительность - 98,7\%, но худшая специфичность описаны в морфометрической модели [12]. Модели использования нейронных сетей CNN/RNN (Tomita et al., 2018) осуществляют качественную классификацию (наличие/отсутствие перелома) и демонстрируют чувствительность 85,2\% и специфичность 95,8\% [16]. В данной модели анализировались только срединные срезы (5\% от всего 3D-массива), что ограничивало ее использование при неточной укладке, вынужденном положении пациента или выраженном сколиозе.
Полученные нами данные AUC ROC для отдельных позвонков/пациентов, в том числе для отложенной, тестовой выборки: чувствительность 97,8\%; специфичность 95,6\%, сопоставимы с данными Nicolaes at al., 2019 (чувствительность 95\%; специфичность 93\%) [11], полученными при кросс-валидации (5 выборок из 90 пациентов). В исследовании Tomita et al. [16], ROC AUC от 0,903 до 0,918 в зависимости от реализованной CNN модели, проводилось тестирование на отложенной выборке 129 пациентов. В нашем исследовании отложенная тестовая выборка пациентов также не использовалась для обучения нейросети.

B pаботе Antonio et al. [9] нейронная сеть для детекции КП была обучена всего на 15 КТ-сериях, точность на тестовой выборке составила 93,29\%, однако небольшой объем данных мог привести к переобучению модели.

B нашей работе ROC AUC для отложенной выборки (60 пациентов) близка к результатам, полученным при кросс-валидации (100 пациентов). Это говорит об отсутствии переобучения нейронных сетей, входящих в модель Comprise-G.

Полученные нами данные о бимодальном распределении переломов тел позвонков с максимумами на среднегрудном (вершина грудного кифоза) и верхнепоясничном отделах позвоночника (максимальные осевые нагрузки) соответствуют данным Burns et al., 2017 [12], Nicolaes at al., 2019 [11].

Предполагаются дальнейшие исследования по валидации разработанной модели Comprise-G. Перспективой внедрения данного подхода является проведение оппортунистического скрининга остеопороза при выполнении КТ ОГК и брюшной полости.

Ограничения исследования

Наиболее вероятные ограничения модели ИИ Comprise-G для автоматической диагностики КП: сканирование других анатомических регионов, на других томографах или с другими условиями (фильтр реконструкции, иная толщина среза и др.), сканирование пациентов с металлическими имплантами, сканирование пациентов с повышенным ИМТ (снижение отношения сигнал/шум) либо применение итеративных механизмов шумоподавления или сканирования со сниженной лучевой нагрузкой.

Учитывая методический характер данной статьи, мы предполагаем более широкое доклиническое тестирование данной модели в различных условиях для уточнения реальных ограничений и выработки более детальных методических указаний по использованию модели Comprise-G.

\section{ЗАКЛЮЧЕНИЕ}

Разработанная модель Comprise-G при первичном анализе позволяет с высокой эффективностью диагностировать КП тел позвонков по данным КТ-сканирования ОГК. Модель представляет собой последовательность двух сверточных нейронных сетей, осуществляющих поиск позвоночника, его «выпрямление» и морфометрию отдельных позвонков - определение вентрального, срединного и дорсального размеров с вычислением индекса компрессии и последующей классификацией в соответствии с полуколичественной шкалой Genant. 
Для обучения модели использованы анонимизированные КТ-изображения 100 пациентов из нескольких медицинских организаций. Разметка проводилась 7 разметчиками со средней относительной погрешностью 3\% под контролем двух рентгенологов со стажем 15 и 35 лет, тестирование работы модели Comprise-G проводилось как при кросс-валидационных данных, так и на отложенной тестовой выборке из 60 пациентов, не использованной при обучении модели. При тестировании на отложенной выборке ROC AUC, для клинически значимых переломов с компрессией более 25\%: AUC 0.978/0.956 (позвонки/ пациенты).

\section{ДОПОЛНИТЕЛЬНАЯ ИНФОРМАЦИЯ}

Источник финансирования. Работа и подготовка статьи проведены на личные средства авторского коллектива.

Конфликт интересов. Авторы декларируют отсутствие явных и потенциальных конфликтов интересов, связанных с публикацией настоящей статьи.

Участие авторов. Петряйкин А.В. - разработка дизайна исследования, общее руководство группой разметчиков, валидация разметки; Белая Ж.Е. - постановка задачи, контроль этапов выполнения работы; Киселева А.Н. - статистический анализ, экспертная разметка; Артюкова 3.Р. - выгрузка данных, экспертная разметка, макетирование рукописи; Беляев М.Г. - контроль взаимодействия группы разработчиков модели, взаимодействие с клинической группой; Кондратенко В.А. - разработка II этапа работы модели; Писов М.Е. - разработка I этапа работы модели, разработка аннотатора; Соловьёв А.В. - экспертная разметка; Сморчкова А.К. - экспертная разметка, разработка внутренней документации по разметке; Абуладзе Л.Р. - экспертная разметка; Киева И.Н. - экспертная разметка; Феданов В.А. - экспертная разметка; Яссин Л.Р. - экспертная разметка; Семенов Д.С. - анонимизация данных; Кудрявцев Н.Д. анализ и выгрузка данных; Щелыкалина С.П. - статистический анализ; Зинченко В.В. - оформление публикации, организация внутреннего согласования проекта; Ахмад Е.С. - техническое IT-обеспечение работы группы разметчиков, обеспечение безопасного перемещения данных; Сергунова К.А. - организация регистрации анонимизированных данных, разработка документации по разметке; Гомболевский В.А. - разработка дизайна модели; Низовцова Л.А. - валидация разметки, разработка документации по разметке; Владзимирский А.В. - разработка дизайна исследования, организация этапных коллоквиумов по этапам выполнения работы; Морозов С.П. - анализ и редактирование макета рукописи, общее руководство выполняемым исследованием. Все авторы внесли значимый вклад в проведение исследования и подготовку статьи, прочли и одобрили финальную версию статьи перед публикацией.

Благодарности. Авторы выносят благодарность отделу координации научной деятельности ГБУЗ «НПКЦ ДиТ ДЗМ» за помощь на всех этапах научной работы.

\section{СПИСОК ЛИТЕРАТУРЫ| REFERENCES}

1. Мельниченко Г.А., Белая Ж.Е., Рожинская Л.Я., и др. Федеральные клинические рекомендации по диагностике, лечению и профилактике остеопороза // Проблемы эндокринологии. 2017. - T. 63. — №6. - C. 392-426. [Mel’nichenko GA, Belaya ZhE, Rozhinskaya LYa, et al. Russian federal clinical guidelines on the diagnostics, treatment, and prevention of osteoporosis. Problemy endocrinologii. 2017;63(6):392-426. (in Russ.)]. doi: 10.14341/probl2017636392-426

2. Лесняк О.М., Баранова И.А., Белова К.Ю. и др. Остеопороз в Российской Федерации: эпидемиология, медико-социальные и экономические аспекты проблемы (обзор литературы) // Травматология и ортопедия России. - 2018. - Т. 24. - №1. C. 155-168. [Lesnyak OM, Baranova IA, Belova KYu et al. Osteoporosis in Russian Federation: Epidemiology, Socio-Medical and Economical Aspects (Review). Travmatologiya i ortopediya Rossii [Traumatology and orthopedics of Russia]. 2018;24(1):155-168. (in Russ.)]. doi: 10.21823/2311-2905-2018-24-1-155-168

3. Лесняк О.М. Аудит состояния проблемы остеопороза в странах восточной европы и центральной азии 2010 // Остеопороз и остеопатии. - 2011. - T. 14. - №2. - C. 3-6. [Lesnyak OM. The Eastern European \& Central Asian Regional Audit Epidemiology, costs \& burden of osteoporosis in 2010. Osteoporosis and bone diseases. 2011;14(2):3-6. (in Russ.)]. doi: 10.14341/osteo201123-6

4. Kanis JA, McCloskey EV, Johansson H, et al. European guidance for the diagnosis and management of osteoporosis in postmenopausal women. Osteoporosis International. 2013; 24:23-57. doi: 10.1007/s00198-012-2074-y

5. Williams AL, Al-Busaidi A, Sparrow PJ, et al. Underreporting of osteoporotic vertebral fractures on computed tomography. European journal of radiology. 2009;69(1):179-183. doi: 10.1016/j.ejrad.2007.08.028

6. Carberry GA, Pooler BD, Binkley N, et al. Unreported vertebral body compression fractures at abdominal multidetector CT. Radiology. 2013;268(1):120-126. doi: 10.1148/radiol.13121632

7. Black DM, Arden NK, Palermo L, et al. Prevalent vertebral deformities predict hip fractures and new vertebral deformities but not wrist fractures. J. Bone Miner. Res. 1999;14(5): 821-828. doi: 10.1359/jbmr.1999.14.5.821

8. Ahmed N, Mandel R, Fain MJ. Frailty: An Emerging Geriatric Syndrome. The American Journal of Medicine. 2007;120(9):748-753. doi: 10.1016/j.amjmed.2006.10.018
9. Antonio CB, Bautista LGC, Labao AB et al. Vertebra fracture classification from 3D CT lumbar spine segmentation masks using a convolutional neural network. 10th Asian Conference on Intelligent Information and Database Systems, ACIIDS 2018. 2018 March 19-21. Dong Hoi City, Vietnam;449-458. doi: 10.1007/978-3-319-75420-8_43

10. Valentinitsch A, Trebeschi S, Kaesmacher J, et al. Opportunistic osteoporosis screening in multi-detector ct images via local classification of textures. Osteoporosis international. 2019;30(6):1275-1285. doi: 10.1007/s00198-019-04910-1

11. Nicolaes J, Raeymaeckers S, Robben D, et al. Detection of vertebral fractures in CT using 3D Convolutional Neural Networks. Computational Methods and Clinical Applications for Spine Imaging. 2019:3-14. doi: 10.1007/978-3-030-39752-4_1

12. Burns JE, Yao J, Summers RM. Vertebral body compression fractures and bone density: automated detection and classification on CT images. Radiology. 2017;284(3):788-797. doi: 10.1148/radiol.2017162100

13. Roth HR, Wang Y, Yao J, et al. Deep convolutional networks for automated detection of posterior-element fractures on spine CT. Medical Imaging 2016: Computer-Aided Diagnosis. 2016. doi: 10.1117/12.2217146

14. Bar A, Wolf L, Amitai OB, et al. Compression fractures detection on CT. Medical Imaging 2017: Computer-Aided Diagnosis. 2017. doi:10.1117/12.2249635

15. Tomita N, Cheung YY, Hassanpour S. Deep neural networks for automatic detection of osteoporotic vertebral fractures on CT scans. Computers in biology and medicine. 2018;(98);8-15. doi: 10.1016/j.compbiomed.2018.05.011

16. Genant HK, Wu CY, Van KC, et al. Vertebral fracture assessment using a semiquantitative technique. Journal of bone and mineral research. 1993;8(9):1137-1148. doi: 10.1002/jbmr.5650080915

17. Указ Президента РФ от 10 октября 2019 г. № 490 «О развитии искусственного интеллекта в Российской Федерации». [Decree of the President of Russian Federation N490 of 10 October 2019. O razvitii iskusstvennogo intellekta v Rossiiskoi Federatsii». (In Russ.)]. Доступно по: http://www.kremlin.ru/acts/bank/44731. Ссылка активна на 03.09.2020.

18. Adams JE, Lenchik L, Roux C, Genant HK. Vertebral Fracture Initiative. Part II. Radiological Assessment of Vertebral Fracture. [Internet]. [cited 2020 Aug 5]; 49 p. Available from: https://www.iofbonehealth.org/ sites/default/files/PDFs/Vertebral\%20Fracture\%20Initiative/IOF_VFIPart_II-Manuscript.pdf 
19. Alqahtani FF, Offiah AC. Diagnosis of osteoporotic vertebral fractures in children. Pediatric Radiology. 2019;49(3);283-296. doi: 10.1007/s00247-018-4279-5

20. Ronneberger O, Fischer P, Brox T. U-net: Convolutional networks for biomedical image segmentation. Medical Image Computing and Computer-Assisted Intervention MICCAI 2015. 2015 October 4-9. Munich, Germany; 234-241. doi: 10.1007/978-3-319-24574-4_28

21. Kingma DP, Ba JL. Adam: A method for stochastic optimization. 3rd International Conference on Learning Representations, ICLR 2015 Conference Track Proceedings [Internet]. 2015 May 7-9. San Diego, USA. Available from: https://arxiv.org/pdf/1412.6980.pdf

22. Redmon J, Farhadi A. Yolov3: An incremental improvement. [Internet]. [cited 2020 Jul 20]; 6 p. Available from: https://arxiv.org/pdf/1804.02767.pdf

23. Pisov M, Kondratenko V, Zakharov A et al. Keypoints localization for joint vertebra detection and fracture severity quantification. [Internet]. [cited 2020 Jul 20]: 13 p. Available from: https://arxiv.org/pdf/2005.11960.pdf

24. Derkatch S, Kirby C, Kimelman D, Jozani MJ, et al. Identification of Vertebral Fractures by Convolutional Neural Networks to Predict Nonvertebral and Hip Fractures: A Registry-based Cohort Study of Dual X-ray Absorptiometry. Radiology. 2019;293(2):190-201. doi: 10.1148/radiol.2019190201

25. Kim DH, MacKinnon T. Artificial intelligence in fracture detection: transfer learning from deep convolutional neural networks. Clinical Radiology. 2018;73(5):439-445. doi: 10.1016/j.crad.2017.11.015

26. Pickhardt PJ, Pooler BD, Lauder T, et al. Opportunistic Screening for Osteoporosis Using Abdominal Computed Tomography Scans Obtained for Other Indications. Annals of Internal Medicine. 2013;158(8):588-595. doi: 10.7326/0003-4819-158-8-201304160-00003.

27. Alacreu E, Moratal D, Arana E. Opportunistic screening for osteoporosis by routine CT in Southern Europe. Osteoporosis International. 2017;28(3):983-990. doi: 10.1007/s00198-016-3804-3

Рукопись получена: 27.08.2020. Одобрена к публикации: 21.09.2020. Опубликована online: 26.10.2020.

\section{ИНФОРМАЦИЯ ОБ АВТОРАХ [AUTHORS INFO]}

*Артюкова Злата Романовна [Zlata R. Artyukova]; адрес: Россия, 127051, Москва, ул. Петровка, д. 24 [address: 24 Petrovka street, 127051 Moscow, Russia]; ORCID: http://orcid.org/0000-0003-2960-9787; eLibrary SPIN: 5873-2280; e-mail: zl.artyukova@gmail.ru

Петряйкин Алексей Владимирович, к.м.н., доцент [Alexey V. Petraikin, PhD, Med., Associate Professor]; ORCID: http://orcid.org/0000-0003-1694-4682; eLibrary SPIN: 6193-1656; e-mail: alexeypetraikin@gmail.com

Белая Жанна Евгеньевна, д.м.н., професcop [Zhanna E. Belaya, PhD, Professor];

ORCID: https://orcid.org/0000-0002-6674-6441; eLibrary SPIN: 4746-7173; e-mail: jannabelaya@gmail.com

Киселёва Анастасия Николаевна [Anastasia N. Kiseleva]; ORCID: https://orcid.org/0000-0002-5713-5307; eLibrary SPIN: 9586-5720, email: anastasia.kiseleva.97@mail.ru

Беляев Михаил Геннадьевич, к.ф.-м.н. [Mikhail G. Belyaev, PhD]; ORCID: http://orcid.org/0000-0001-9906-6453, eLibrary SPIN: 2406-1772, email: m.belyaev@skoltech.ru

Кондратенко Владимир Анатольевич [Vladimir A. Kondratenko]; ORCID: http://orcid.org/0000-0001-8123-0462; eLibrary SPIN: 9265-9820; email: v.kondratenko@skoltech.ru

Писов Максим Евгеньевич [Maxim E. Pisov]; ORCID: http://orcid.org/0000-0001-8727-5792; eLibrary SPIN: 7812-9031; email:m.pisov@skoltech.ru

Соловьев Александр Владимирович [Alexander V. Solovev]; ORCID: http://orcid.org/0000-0003-4485-2638; eLibrary SPIN: 9654-4005; email: atlantis.92@mail.ru

Сморчкова Анастасия Кирилловна [Anastasia K. Smorchkova]; ORCID: http://orcid.org/0000-0002-9766-3390; eLibrary SPIN: 4345-8568, email: smanki97@gmail.com

Абуладзе Лия Руслановна [Liya R. Abuladze]; ORCID: https://orcid.org/0000-0001-6745-1672; eLibrary SPIN: 8640-9989; email: drliaabuladze@gmail.com

Киева Ирина Николаевна [Irina N. Kieva]; ORCID: https://orcid.org/0000-0002-4060-5966; eLibrary SPIN:2279-9141; e-mail: irina.kieva9@yandex.ru

Феданов Владимир Александрович [Vladimir A. Fedanov]; ORCID: https://orcid.org/0000-0003-1979-0359; eLibrary SPIN:4700-0649; e-mail: vfedanov@gmail.com

Яссин Лейла Раедовна [Leila R. lassin]; ORCID: https:/orcid.org/0000-0002-2772-1401; eLibrary SPIN:3439-6381; e-mail: yassin.leila@yandex.ru

Семенов Дмитрий Сергеевич [Dmitry S. Semenov]; ORCID:http://orcid.org/0000-0002-4293-2514; eLibrary SPIN: 2278-7290; e-mail: d.semenov@npcmr.ru

Кудрявцев Никита Дмитриевич [Nikita D.Kudryavtsev]; ORCID: https://orcid.org/0000-0003-4203-0630; eLibrary SPIN: 1125-8637; e-mail: n.kudryavtsev@npcmr.ru

Щелыкалина Светлана Павловна, к.М.н., доцент [Svetlana P. Shchelykalina, PhD, Med., Associate Professor];

ORCID: https://orcid.org/0000-0003-3292-8949; eLibrary SPIN: 9804-0820; e-mail: svetlanath@gmail.com

Зинченко Виктория Валерьевна [Victoria V. Zinchenko]; ORCID: https://orcid.org/0000-0003-3292-8949;

eLibrary SPIN: 4188-0635; e-mail: v.zinchenko@npcmr.ru

Ахмад Екатерина Сергеевна [Ekaterina S. Akhmad]; ORCID: https://orcid.org/0000-0002-8235-936;

eLibrary SPIN: 5891-4384; e-mail: e.ahmad@npcmr.ru

Сергунова Кристина Анатольевна, к.т.н. [Kristina A. Sergunova, PhD]; ORCID: http://orcid.org/0000-0002-9596-7278; eLibrary SPIN: 6946-3205; e-mail: sergunova@npcmr.ru

Гомболевский Виктор Александрович, к.M.H. [Victor A. Gombolevsky, MD, PhD];

ORCID: http://orcid.org/0000-0003-1816-1315; eLibrary SPIN: 6810-3279; e-mail: gombolevskiy@npcmr.ru 
Низовцова Людмила Арсеньевна, д.м.н., профессор [Lyudmila A. Nisovstova, PhD, Professor]; ORCID: https:// orcid.org/0000-0002-9614-450, eLibrary SPIN: 9957-8107; e-mail: nizovtsova@npcmr.ru

Владзимирский Антон Вячеславович, д.м.н. [Anton V. Vladzymyrskyy, MD, PhD];

ORCID: http://orcid.org/0000-0002-2990-7736; eLibrary SPIN: 3602-7120; e-mail: a.vladzimirsky@npcmr.ru

Морозов Сергей Павлович, д.м.н., профессор [Sergey P. Morozov, MD, PhD, Professor];

ORCID: http://orcid.org/0000-0001-6545-6170; eLibrary SPIN: 8542-1720; e-mail: morozov@npcmr.ru

\section{ЦИТИРОВАТЬ:}

Петряйкин А.В., Белая Ж.Е., Киселева А.Н., Артюкова З.Р., Беляев М.Г., Кондратенко В.А., Писов М.Е., Соловьев А.В., Сморчкова А.К., Абуладзе Л.Р., Киева И.Н., Феданов В.А., Яссин Л.Р., Семёнов Д.С., Кудрявцев Н.Д., Щелыкалина С.П., Зинченко В.В., Ахмад Е.С., Сергунова К.А., Гомболевский В.А., Низовцова Л.А., Владзимирский А.В., Морозов С.П. Технология искусственного интеллекта для распознавания компрессионных переломов позвонков с помощью модели морфометрического анализа, основанной на сверточных нейронных сетях // Проблемы эндокринологии. 2020. — T. 66. — №5. — C. 48-60. doi: https://doi.org/10.14341/probl12605

\section{TO CITE THIS ARTICLE:}

Petraikin AV, Belaya ZhE, Kiseleva AN, Artyukova ZR, Belyaev MG, Kondratenko VA, Pisov ME, Solovev AV, Smorchkova AK, Abuladze AR, Kieva IN, Fedanov VA, lassin LR, Semenov DS, Kudryavtsev ND, Shchelykalina SP, Zinchenko VV, Akhmad ES, Sergunova KA, Gombolevskiy VA, Nisovtsova LA, Vladzymyrskyy AV, Morozov SP. Artificial intelligence for diagnosis of vertebral compression fractures using a morphometric analysis model, based on convolutional neural networks. Problems of Endocrinology. 2020;66(5):48-60. doi: https://doiorg/1014341/probl12605 